\title{
Effects of Static Apnea Training on Pulmonary Function, Blood Lactate Response and Exercise Performance of Elite Swimmers
}

\author{
Heejeong Son ${ }^{1} \mathrm{PhD}$, Yunah Jeon ${ }^{2} \mathrm{PhD}$, Hyosik Kim ${ }^{3} \mathrm{PhD}$ \\ ${ }^{1}$ Department of Center for Sports Science, Gyeonggi-do Sports Council, Suwon; ${ }^{2}$ Department of Health and Rehabilitation, Yeoju Institute of Technology, Yeoju; \\ ${ }^{3}$ Department of Physical Education, Korea National Sport University, Seoul, Korea
}

PURPOSE: The purpose of this study was to investigate the effects of training on improvement in pulmonary function and swimming performance by implementing static apnea training for 4 weeks in elite male swimmers.

METHODS: Twenty elite swimmers were divided into two groups, the apnea training group (Apnea, $\mathrm{n}=11$ ) and the control group (control, $n=9$ ). Each swimmer received identical swimming and ground training for 4 weeks. The apnea group performed 10 sets of breathholding a day additionally. In all participants, the pulmonary function tests [forced vital capacity (FVC) and forced expiratory volume in 1 second (FEV1)] were evaluated. The blood lactate level was also measured after each swimmer completed swimming 5 laps.

RESULTS: After 4 weeks of static apnea training, the FVC of the apnea group significantly increased ( $p=.008)$, whereas the FEV1/FVC ratio did not change. Breath-holding time increased significantly after apnea training $(p=.001)$. There were no significant differences between the groups in the 50-m time trial records. However, the apnea group show a significant difference at the 4 th $(p=.013)$ and 5 th trial $(p=.023)$. The blood lactate levels after the $50-\mathrm{m}$ trial was not significantly different between the groups, but the levels in the apnea group showed a significant increase in the results of the 2 nd trial compared to those before apnea training $(p=.008)$.

CONCLUSIONS: The results of our study reveal that static apnea training improves pulmonary function, in contrast to a few time trials that show varying differences in swimming records and blood lactate levels. These results warrant a review of the training protocol to evaluate the effect on performance. In conclusion, static apnea training is a potential exercise that can improve performance in competitive sports.

Key words: Swimmer, Apnea training, FVC, FEV1/FVC, Blood lactate

\section{INTRODUCTION}

Training regimes of elite swimmers require systematic performance improvement plans and should avoid overtraining based on the principles stated in sports science. Regulation of inspiratory and expiratory muscles while breathing is a prerequisite for maintaining an efficient stroke technique while swimming. This is because the demand to control the breathing pattern in competitive swimming is greater than in other athletics events [1]. Swimming improves the function of inspiratory muscles [2]. However, special inspiratory muscle training enhances not only strength [3-5], but also endurance. It is known to improve the athletic performance in athletes [6,7]. Swimmers performed apneas during regular training. This strengthened both expiratory and inspiratory muscles and thus improved pulmonary function [8].

Apnea induces a cardiovascular adaptation called the diving response $[9,10]$, which lessens the cardiac output by reducing the stroke volume and heart rate (HR) [11-13]. The diving response is known to be enhanced by various factors such as face immersion in low temperature and in low oxygen environments [14]. A study conducted in underwater athletes showed that even though the HR increased, interspersed epi-

Corresponding author: Hyosik Kim Tel +82-2-410-6875 Fax+82-2-410-6875 E-mail hyosikaknsu.ac.kr

Received 20 Jun 2020 Revised 14 Aug 2020 Accepted 19 Aug 2020

(a) This is an Open Access article distributed under the terms of the Creative Commons Attribution Non-Commercial License (https://creativecommons.org/licenses/by-nc/4.0/) which permits unrestricted non-commercial use, distribution, and reproduction in any medium, provided the original work is properly cited. 
sodes of bradycardia were observed during dynamic apnea in synchronized swimmers [15] and trained breath-holding divers [16]. Bradycardic response or arterial oxygen desaturation was reported to be related to the performance of elite synchronized swimmers, divers, and swimmers $[15,17,18]$.

A study carried out on trained breath-holding divers [17] showed that arterial oxygen desaturation occurred faster accompanied by an increase in lactate during dynamic dives than in static dives. Arterial oxygen desaturation and plasma lactate, which increase during apnea, indicate that they are highly dependent on the anaerobic metabolism $[9,11,19,20]$. Lactate is considered important in short-distance swimming [21], such as 50-m and 100-m, as they are most dependent on the anaerobic metabolism for ATP re-synthesis [21-24]. This is cited in recent research papers, in which lactate measurement along with the performance of swimmers is frequently applied as a means to evaluate the achievement of training $[21,25,26]$.

Repeated apnea training induces hypoxemia in the spleen and kidney, increasing hematocrit (Hct) and hemoglobin ( $\mathrm{Hb})$ [27,28]. A sharp decrease in the level of tissue oxygen during apnea stimulates erythropoietin (EPO) production, which promotes the proliferation and maturation of red blood cell precursors in the bone marrow and eventually increases oxygen delivery to the muscles, and improving sports performance. Trained breath-holding divers showed significantly higher $\mathrm{Hb}$ during apnea than untrained divers and elite cross-country skiers [28], and revealed a $24 \%$ increase in EPO concentration caused by repeated apneas [27]. It was proposed that physiological adaptation by apnea training may be an effective alternative to hypobaric or normobaric hypoxia [29]. Thus, apnea training is being offered to improve the performance of athletes in competitive sports where aerobic and/or anoxic performance is important.

Most of the previous studies have been conducted in synchronized swimmers [15], breath-holding divers $[14,16,17,19]$ and underwater hockey players [18] to analyze their physiological responses to acute apnea; or in swimmers to investigate the presence of physiological indicators of one-time dynamic apnea during swimming, focusing specifically on their performance. The only study to examine the physiological response index and exercise performance after apnea training for swimmers [8] comprised a small sample size and had no control group. It is therefore difficult to explain whether the effect was due to training alone or both swimming and apnea training.

The possibility that the physiological adaptation reaction caused by apnea training may be an effective training method for elite swimmers was considered. However, it necessitated newer attempts to scientifically verify the same for development of a systematic training program based on evidence. Therefore, in our study, we included 20 male elite swimmers who were divided into a 'control' group that continued with the existing training method and an 'apnea' group that performed apnea training in addition to the existing training method. Records of swimming performance and physiological variables (pulmonary function, lactic acid) were analyzed for verifying the effects.

\section{METHODS}

\section{Participants}

The participants included 20 male swimmers with an average experience of 10 years ( $10 \pm 1.3$ years) at H University. All athletes who participated in the study were elite national level swimmers who had placed 3rd in competitions. These athletes stayed in the same dormitory, received the same diet, swam and trained regularly (average $15 \pm 0.03$ hours/week).

The study was conducted in conformance with the principles laid down in the Declaration of Helsinki. Participants provided informed consent. After obtaining history about previous injuries and medications, 20 male swimmers were finally selected. They were divided into 2 groups. One group comprised participants who continued with the existing training method (Control, $\mathrm{n}=9$ ) and the other group performed static apnea training in addition to the existing training method (Apnea, $\mathrm{n}=11$ ) The physical characteristics of the participants are presented in Table 1.

\section{Experimental methods}

The experiment was conducted both in the H University swimming pool in Seoul (with an ambient room temperature of $25 \pm 1^{\circ} \mathrm{C}$, and water temperature of $27 \pm 1^{\circ} \mathrm{C}$ ) and in the laboratory. Each swimmer consumed the same diet and underwent similar training programs including the amount of swimming and ground training over a period of four weeks. Table 2 shows the content and duration of training in the two groups.

Table 1. Characteristics of subjects

\begin{tabular}{lcc}
\hline & Control $(\mathrm{n}=9)$ & Apnea $(\mathrm{n}=11)$ \\
\hline Age $(\mathrm{yr})$ & $21.56 \pm 1.13$ & $21.50 \pm 1.08$ \\
Height $(\mathrm{cm})$ & $181.67 \pm 4.27$ & $179.90 \pm 4.41$ \\
Body weight $(\mathrm{kg})$ & $77.56 \pm 6.54$ & $74.20 \pm 3.77$ \\
Body mass index $\left(\mathrm{kg} / \mathrm{m}^{2}\right)$ & $23.51 \pm 2.08$ & $22.95 \pm 1.35$ \\
\hline
\end{tabular}


Table 2. Training program

\begin{tabular}{|c|c|c|c|c|c|c|c|}
\hline & & Monday & Tuesday & Wednesday & Thursday & Friday & Saturday \\
\hline \multirow{2}{*}{ Swim training } & $\operatorname{Am}(2 \mathrm{hr})$ & $7,000 \mathrm{~m}$ & Rest & Rest & Rest & $7,000 \mathrm{~m}$ & $7,500 \mathrm{~m}$ \\
\hline & $\mathrm{Pm}(3 \mathrm{hr})$ & $8,500 \mathrm{~m}$ & $9,000 \mathrm{~m}$ & $9,000 \mathrm{~m}$ & $8,500 \mathrm{~m}$ & $9,000 \mathrm{~m}$ & Rest \\
\hline \multirow[t]{7}{*}{ Dry-landing training } & Monday, Wednesday, Friday (1 hr) & & & \multicolumn{3}{|c|}{ Squat $1 \mathrm{RM} 80 \%$ 10-12 reps } & 3 min rest between a set \\
\hline & & & & \multicolumn{3}{|c|}{ Chest curl 1 RM 80\% 10-12 reps } & \\
\hline & & & & \multicolumn{3}{|c|}{ Bench press 1 RM $80 \%$ 10-12 reps } & \\
\hline & & & & \multicolumn{3}{|c|}{ Barbell $15 \mathrm{~kg} 3$ sets } & \\
\hline & & & & \multicolumn{3}{|c|}{ Physio ball 3 sets } & \\
\hline & & & & \multicolumn{3}{|c|}{ Medicine ball $5 \mathrm{~kg} 3$ sets } & \\
\hline & & & & \multicolumn{3}{|c|}{ Band training 5 sets } & \\
\hline
\end{tabular}

The apnea training group performed 10 sets of breath-holding a day in addition to their regular training (with two minutes of rest between the sets) and breath-holding times were recorded and analyzed. During the 48 hours before the experiment, participants were restricted from performing too much exercise. Intake of caffeine and alcohol was restricted. The trials were conducted three times to eliminate psychological anxiety and to help the athletes adapt to the experiment.

The interval between experiments for each intervention was not less than 3 days and no more than 7 days, and the experiment was conducted at the same time frame between 3 and 6 pm, for all. Height and weight were measured after arriving at the test site on the day of the experiment. The experimental method, measurement records, and methods of analysis of the physiological variables were executed as follows.

\section{1) Static apnea training}

The maximum period of apnea was measured and recorded. It was defined as the duration from when the participant inhaled maximal air and blocked the nose until the next inhale. The static apnea training was conducted 5 times within 1 hour after waking up, and 5 times before bedtime at night ( 2 minutes rest per session). Apnea training was conducted 10 times per day for 4 weeks.

\section{2) Measurement of physiological variables}

(1) Vital capacity

The vital capacity was measured using the pulmonary function analyzer before and after static apnea training. The maximum forced vital capacity (FVC, L) and the ratio of forced expiration volume for 1 second to the forced vital capacity (FEV1/FVC, \%) were calculated.

\section{(2) Swim record}

Before measuring the maximum power swimming record, 15 minutes of freestyle warm-up and 5 minutes of rest (total 20 minutes) were allocated. Swim paddles (Speedo, Korea; $12 \times 15 \mathrm{~cm}$ ) and swim pins (Spurt, Korea; short fin, shoe size + fin $<5 \mathrm{~cm}$ ) that fit both the hands and feet were worn for performing the measurements. The maximum swimming record of all participants in the 50-m trial was measured using a stopwatch (Seiko). The 50-m distance was traversed at maximum power 5 times using a freestyle technique and starting from the water edge to reduce the starting error due to a dive start.

\section{(3) Blood lactate concentration}

The blood lactate concentration was measured by collecting a small amount of blood at the fingertips using the Lancet (TechLet, Du Yee Chemical, Korea), which was then used for analysis in a lactate analyzer (YSI 1500, YSI, USA) at rest and at an interval of $50 \mathrm{~m}$.

\section{Statistical analyses}

For all results, we used the average of the two values that was measured. The SPSS/PC+Version 18.0 program was used for analysis. Twoway ANOVA with repeated measures was performed to analyze the difference between related indicators depending on whether apnea training was performed (apnea vs. control), and the least significant difference (LSD) method was applied for the post-hoc test. A paired $t$-test was conducted to verify the differences between related indicators before and after apnea training. The statistical significance level was set at $p=.05$.

\section{RESULTS}

\section{Changes in the FVC values after 4 weeks of static apnea training}

After 4 weeks of apnea training, the FVC showed a significant interaction over time in the group $(p=.001)$. In the control group, there was no 


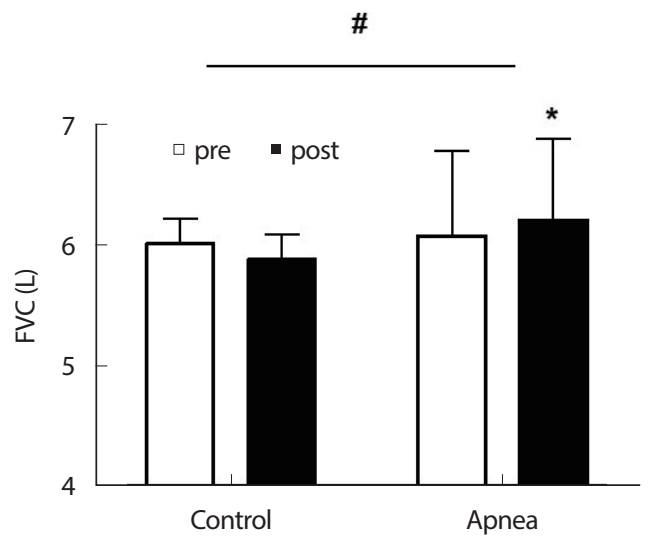

Fig. 1. Changes in the forced vital capacity values after 4 weeks of static apnea training. ${ }^{*} p<.05$ time $\times$ group interaction, ${ }^{*} p<.05$ between pre and post values.

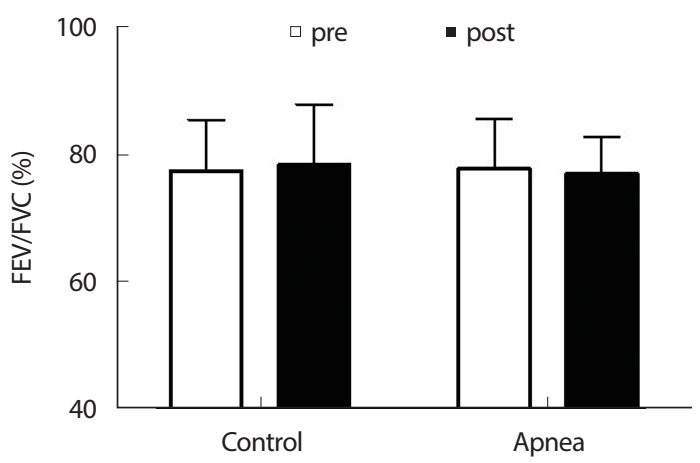

Fig. 2. Changes in the FEV/FVC ratio after 4 weeks of static apnea training.

difference between the values obtained before and after the training ( $p>.05)$. However, in the apnea group the values of FVC increased significantly after training $(p=.008)$ (Fig. 1).

\section{Changes in the FEV1/FVC (\%) ratio after 4 weeks of static apnea training}

After 4 weeks of apnea training, the FEV1/FVC ratio revealed no interaction between the groups, and neither the control group nor the apnea group showed any significant variations before and after training (Fig. 2).

\section{Changes in the breath-holding time during static apnea training for 4 weeks}

The breath-holding time in the 4 weeks showed interactions with group and time ( $p=.036)$. The result of the post-hoc analysis, divulged no differences between the groups, but the time of breath-holding had increased significantly with time due to apnea training $(p<.05)$. In the control group the duration of breath-holding increased significantly in

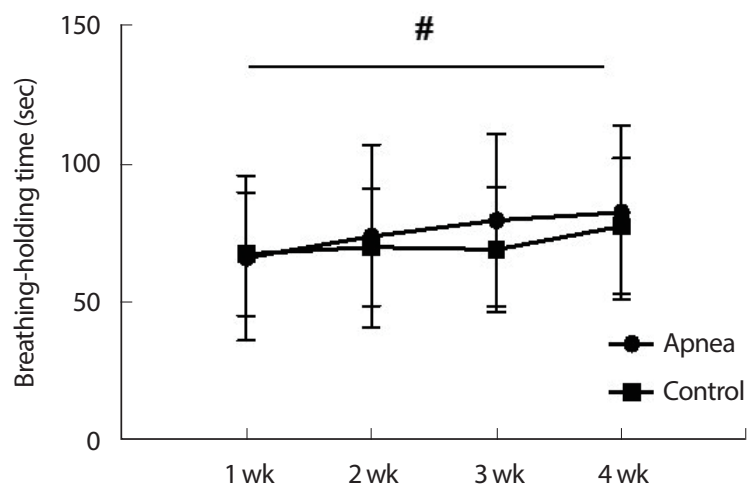

Fig. 3. Changes in the time of breathing-holding during static apnea training. ${ }^{*} p<.05$ time $\times$ group interaction.

the 2 nd week $(p=.020)$, the 3 rd week $(p=.001)$ and the 4 th week $(p=$ .001) compared to the 1st week. In the apnea training group, there was a significant increase in the 2nd week ( $p=.001)$, 3rd week $(p=.001)$ and 4 th week ( $p=.001$ ) compared to the 1st week (Fig. 3).

\section{Changes in $50 \mathrm{~m}$ repetitive trial records after 4 weeks of static apnea training}

No remarkable differences were noted between the groups $(p>.05)$ or among the periods ( $p>05)$ in the records following 5 repetitive 50-m trial sessions after 4 weeks of apnea training. Additionally, there was no interaction between the groups or over time ( $p=.772)$. The results in the apnea group showed significant improvement during the 4th $(p=.013)$ and the 5th ( $p=.023)$ trial sessions compared to those before apnea training. In contrast, in the control group, only the 5th trial session showed significant improvement in results compared to those before training $(p=.022)$ (Fig. 4$)$.

\section{Changes in blood lactate concentration after 4 weeks of static apnea training}

After 4 weeks of apnea training, the lactate concentration following 50-m repetitive trial sessions disclosed no differences between the groups ( $p=.856$ ), but variations over time were observed $(p<.01)$. Also, there was no interaction between the groups or over time $(p=.649)$. Lactate concentration following repeated 50-m trial sessions, before and after apnea training for 4 weeks was significantly higher than before apnea training during the 2 nd trial in the apnea group $(p=.008)$. On the other hand, in the control group, there was no difference before and after training $(p>05)$ (Fig. 5). 

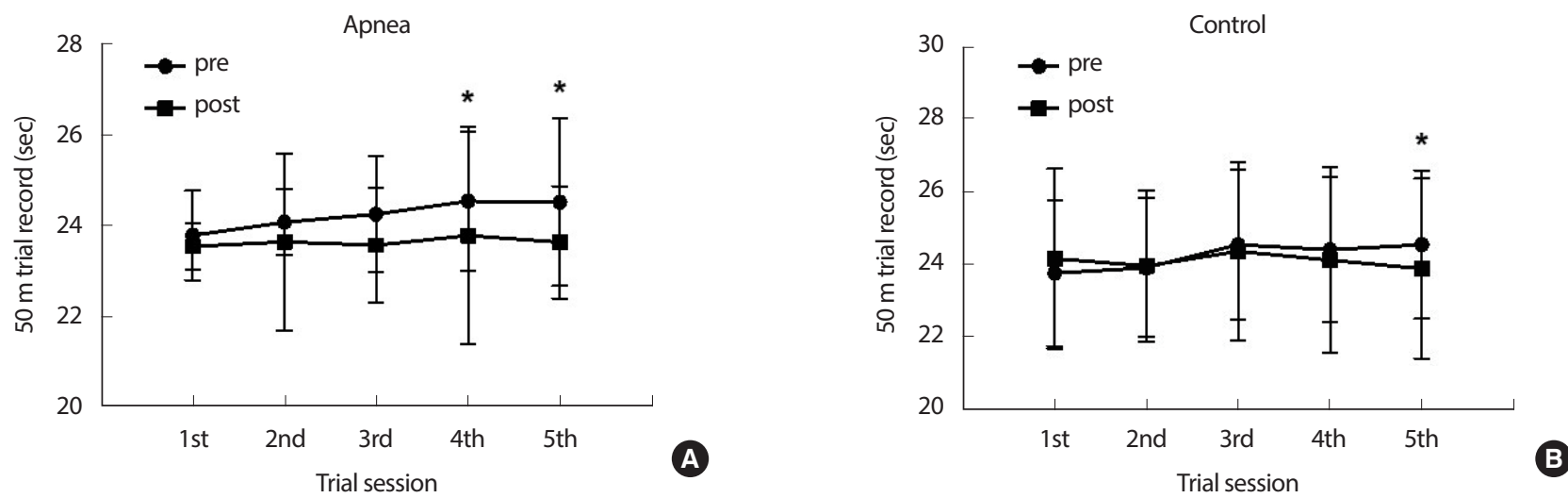

Fig. 4. Changes in 50m records after 4 weeks of static apnea training. (A) The differences in the results pre- and post-training in the apnea group. (B) The differences in the results pre- and post-training in the control group. ${ }^{*} p<.05$ between pre and post the $50 \mathrm{~m}$ trial.
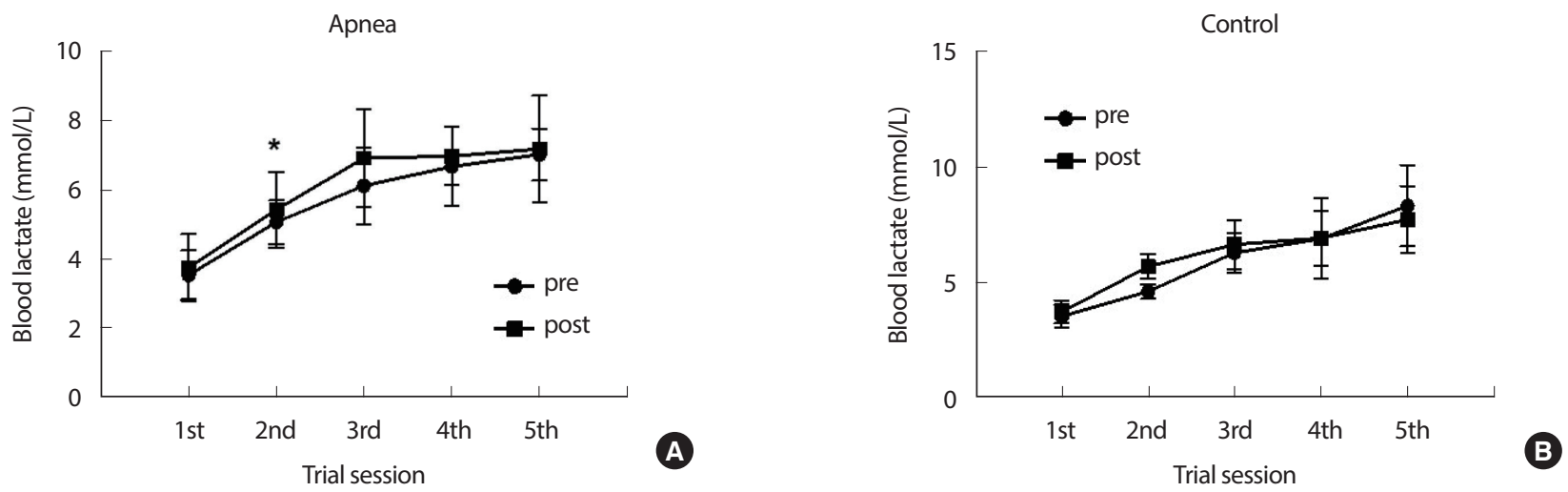

Fig. 5. Changes in blood lactate concentration following 50m record trials after 4 weeks of static apnea training. (A) The differences in lactate concentration pre-and post-training in the apnea group. (B) The differences in lactate concentration pre-and post-training in the control group. ${ }^{*} p<.05$ between pre and post the $50 \mathrm{~m}$ trial

\section{DISCUSSION}

Currently, elite swimmers in Korea undergo high intensity swimming and ground training. The sports instruction field is extremely interested in scientifically-based training methods that can improve the performance without significantly increasing the total amount of training. Thus, we attempted to apply apnea training, known to have a positive effect on breathing patterns, in elite swimmers. The apnea training was performed along with existing training for 4 weeks, and positive changes in pulmonary function in addition to an increase in breath holding time were established. Although no differences were noted between the groups in the 50-m swimming record and lactic acid concentration after apnea training, a significant effect was confirmed in few 50-m trial sessions over time.

The FVC values of the apnea group increased significantly after 4 weeks of static apnea training, while the FEV1/FVC ratio did not change in our study. This result is similar to that reported in the previous study comprising fin swimmers [30] in which improvement in pulmonary function after breath-holding training was attributed to strengthening of the respiratory muscles.

In addition, duration of breath-holding revealed a pertinent increase due to apnea training. There was no significant difference between the groups in the $50 \mathrm{~m}$ trial session recording of 5 repetitions, but a change was perceived in the apnea group in the 4 th and 5 th trial sessions. The control group was observed to have improved records during the 5th trial session. The lactic acid concentration measured during the 50-m trial session was not different between the groups, but the apnea group showed significant increase in the results in the 2nd trial session compared to those obtained before apnea training. These results suggest that static apnea training is an effective intervention method that can increase the pulmonary function and breath-holding time to maximize the performance in swimmers. However, when performing a 50-m repe- 
tition trial, there was no evidence of improvements of the swimming record or a change in lactate production capacity. It is difficult to conclude that the effect of apnea training on exercise performance is large because there were no notable differences between the groups and the impact was minimal at some timepoints. The duration of this study (4 weeks) is short, and the evidence for the appropriateness of the swimming proto$\mathrm{col}(50 \mathrm{~m} \times 5 \mathrm{rep})$ for evaluating exercise performance is limited.

Apnea training has recently been introduced as an effective alternative to hypobaric or normobaric hypoxia exposure by inducing positive adaptive responses such as increased breath-holding time, pulmonary volume [31], bradycardia and vasoconstriction during apnea, and reduced acidosis and oxidative stress [19,32]. In fact, in the case of breathholding divers, chemosensitivity for hypercapnia and hypoxia is increased $[33,34]$. It may cause less ventilation reaction and lessen dyspnea during submaximal exercise. These changes have been suggested to be beneficial to a wide range of sports players, including divers [32].

Swimming is a competitive sport that requires about 4 times more energy than running [35] and requires higher breathing pattern control capabilities than athletics. Therefore, good respiratory muscle performance and pulmonary function are prerequisites for maintaining an efficient swimming stroke technique [1]. Improved pulmonary function is accompanied by improved respiratory muscle performance [36] and prolonged and delayed respiratory muscle fatigue during maximal exercise, [29] which improves exercise performance. In our study, static apnea training for 4 weeks performed on elite swimmers was seen to improve pulmonary function by increasing FVC similar to the results of the study [8] in which an increase in FEV1 was seen after apnea training in swimmers for a longer period (3 months).

In addition, it has been found that it is possible to extend the apnea duration. Repeated apnea training induces splenic contraction, thus reducing the arterial oxygen desaturation [37-39]. Most studies conducted in synchronized swimmers [15], breath-holding divers [14,16,17,19] and underwater hockey players [18] have reported a similar relationship with breath-holding duration as that in players who have already been adapted to dynamic apnea underwater. Previous studies have introduced methods to improve the various factors that can affect performance regardless of where apnea training is performed (underwater or above ground) $[8,30,31]$, although there is a lack of evidence for static apnea training on the ground. Therefore, we aimed to establish a method of improving performance that can be used universally by applying static apnea training on the ground. In accordance with the purpose of this study, static apnea training was conducted for 4 weeks on the ground for elite swimmers, and breath-holding time increased as training continued.

Increase in the duration of apnea while swimming increases resistance to $\mathrm{CO}_{2}$, making it easier to maintain breathing. This minimizes the obstruction caused by breathing to arm coordination and increases stroke length and stroke rate to improve both-arm propulsion [40]. In our study, there were no significant differences between the 50-m trial records in which the 50-m distance was traversed 5 times using the freestyle method, but the apnea group showed improvement in results only in the 4th and 5th trial sessions. In a study that compared the performance of swimmers with apnea and normal frequency breathing, the results revealed that the performance was further improved during normal frequency breathing [10]. However, results of this study were that of one-time dynamic apnea swimming, and it is difficult to directly compare it with the results obtained in our study. In future studies, it would be meaningful to perform long term dynamic and static apnea training and subsequently compare the effects on the performance.

The level of lactic acid in the blood is increased by apnea during exercise, which relies on the anaerobic glycolytic metabolism. This means the accumulation of metabolites in active muscles $[9,41]$. However, training to regulate lactic acid production is a very important factor for swimming ability. A large part of the swimming training is focused on improving the ability to generate lactic acid, which is also used as a means to evaluate the training process and the success of training [21]. Lactic acid concentration is related to muscle lactate concentration and anaerobic exercise performance $[21,22,24,42]$. This has been widely used to evaluate and observe the adaptation of the lactic acid system for energy production due to its relatively simple method of measurement. In our study, the lactic acid concentration measured after 5 repetitions of 50-m swimming did not differ between the groups. However, the apnea group showed significant increase in the 2 nd trial session compared to the results achieved before apnea training. In the case of the dynamic apnea trained breath-holding diver, the arterial oxygen desaturation and lactate increased rapidly at the beginning of exercise [17], whereas in the swimmer, lactatemia was not higher than that achieved during the control condition after acute apnea swimming. This suggests the possibility that the $25 \mathrm{~m}$ is too short to increase the anaerobic glycolytic metabolism or that a late lactatemia peak could occur due to vasoconstriction. As mentioned previously, it may be necessary to review the swimming protocols (distance $\times$ reps) for evaluating the performance following ap- 
nea training in subsequent studies. Apnea temporarily increases the hematocrit, but the effect disappears within 10 minutes of the last apnea $[39,43]$. Thus, a long-term study with repeated apnea training and the potential to cause a physiological metabolic adaptation should be conducted.

Static apnea training for 4 weeks increased the pulmonary function and breath-holding time of elite swimmers, confirming its potential as an exercise mediator to improve athletic performance. However, the effect on athletic performance is not clear, which preempts the identification of the various associated physiological factors. Studies with longterm interventions should be undertaken. The result of our study is meant to provide basic data to the leaders in the field of sports who engage in scientifically based training plans to improve elite swimmers' performance.

\section{CONCLUSIONS}

This study was designed to test the effects of four weeks of static apnea training on the performance of elite swimmers (swimming record) and physiological variables (pulmonary function, lactic acid). According to the results of this study, positive changes in pulmonary function of elite swimmers were observed through static apnea training. Improvement in pulmonary function establishes the potential of this training in strengthening the respiratory muscles and improving performance. Conversely, apnea training did not show a difference between the groups with regard to the swimming record and lactic acid concentration. It was difficult to identify a clear impact on exercise performance because the effect of apnea training was confirmed only in some trial sessions. In the future, it is necessary to examine the long-term interventions and the various physiological factors of apnea training for implementation in the field of competitive sports.

\section{CONFLICT OF INTEREST}

No potential conflict of interest relevant to this article was reported.

\section{ORCID}

$\begin{array}{ll}\text { Hee jeong Son } & \text { https://orcid.org/0000-0002-1360-3201 } \\ \text { Yunah Jeon } & \text { https://orcid.org/0000-0003-4484-4187 } \\ \text { Hyosik Kim } & \text { https://orcid.org/0000-0002-9105-1596 }\end{array}$

\section{AUTHOR CONTRIBUTIONS}

Data curation: H Son, Y Jeon; Formal analysis: Y Jeon; Visualization: Y Jeon; Writing-original draft: H Son, H Kim; Writing-review \& editing: H Son, H Kim

\section{REFERENCES}

1. Kilding AE, Brown S, McConnell AK. Inspiratory muscle training improves 100 and $200 \mathrm{~m}$ swimming performance. Eur J Appl Physiol. 2010;108(3):505-11.

2. Mickleborough TD, Stager JM, Chatham K, Lindley MR, Ionescu AA. Pulmonary adaptations to swim and inspiratory muscle training. Eur J Appl Physiol. 2008;103(6):635-46.

3. Edwards AM, Cooke CB. Oxygen uptake kinetics and maximal aerobic power are unaffected by inspiratory muscle training in healthy subjects where time to exhaustion is extended. Eur J Appl Physiol. 2004;93(1-2):139-44.

4. Gething AD, Williams M, Davies B. Inspiratory resistive loading improves cycling capacity: a placebo controlled trial. Br J Sports Med. 2004;38(6):730-6.

5. Sheel AW. Respiratory muscle training in healthy individuals: physiological rationale and implications for exercise performance. Sports Med. 2002;32(9):567-81.

6. Griffiths LA, McConnell AK. The influence of inspiratory and expiratory muscle training upon rowing performance. Eur J Appl Physiol. 2007;99(5):457-66.

7. Johnson MA, Sharpe GR, Brown PI. Inspiratory muscle training improves cycling time-trial performance and anaerobic work capacity but not critical power. Eur J Appl Physiol. 2007;101(6):761-70.

8. Lemaitre F, Seifert L, Polin D, Juge J, Tourny-Chollet C et al. Apnea training effects on swimming coordination. J Strength Cond Res. 2009;23(6):1909-14.

9. Andersson JP, Linér MH, Fredsted A, Schagatay EK. Cardiovascular and respiratory responses to apneas with and without face immersion in exercising humans. J Appl Physiol. 2004;96(3):1005-10.

10. Guimard A, Prieur F, Zorgati H, Morin D, Lasne F et al. Acute apnea swimming: Metabolic responses and performance. J Strength Cond Res. 2014;28(4):958-63.

11. Foster GE, Sheel AW. The human diving response, its function, and its control. Scand J Med Sci Sports. 2005;15(1):3-12. 
12. Lindholm P, Lundgren CE. The physiology and pathophysiology of human breath-hold diving. J Appl Physiol. 2009;106(1):284-92.

13. Schagatay E, Andersson JP, Nielsen B. Hematological response and diving response during apnea and apnea with face immersion. Eur J Appl Physiol. 2007;101(1):125-32.

14. Andersson JP, Linér MH, Runow E, Schagatay EK. Diving response and arterial oxygen saturation during apnea and exercise in breathhold divers. J Appl Physiol. 2002;93(3):882-6.

15. Rodríguez-Zamora L, Iglesias X, Barrero A, Chaverri D, Erola P, et al. Physiological responses in relation to performance during competition in elite synchronized swimmers. PLoS One. 2012;7(11):e49098.

16. Overgaard K, Friis S, Pedersen RB, Lykkeboe G. Influence of lung volume, glossopharyngeal inhalation and P ET $\mathrm{O}_{2}$ and $\mathrm{P}$ ET $\mathrm{CO}_{2}$ on apnea performance in trained breath-hold divers. Eur J Appl Physiol. 2006;97(2):158-64.

17. Breskovic T, Uglesic L, Zubin P, Kuch B, Kraljevic J, et al. Cardiovascular changes during underwater static and dynamic breath-hold dives in trained divers. J Appl Physiol. 2011;111(3):673-8.

18. Lemaître F, Polin D, Joulia F, Boutry A, Le Pessot D, et al. Physiological responses to repeated apneas in underwater hockey players and controls. Undersea Hyperb Med. 2007;34(6):407.

19. Joulia F, Steinberg JG, Wolff F, Gavarry O, Jammes Y. Reduced oxidative stress and blood lactic acidosis in trained breath-hold human divers. Respir Physiol Neurobiol. 2002;133(1-2):121-30.

20. Palada I, Obad A, Bakovic D, Valic Z, Ivancev V, et al. Cerebral and peripheral hemodynamics and oxygenation during maximal dry breath-holds. Respir Physiol Neurobiol. 2007;157(2-3):374-81.

21. Maglischo EW. Swimming fastest. Human kinetics 2003.

22. Colwin C. Breakthrough swimming. Human kinetics 2002.

23. Gastin PB. Energy system interaction and relative contribution during maximal exercise. Sports Med. 2001;31(10):725-41.

24. Mougios V. Exercise Biochemistry. Champaign, IL: Human Kinetics. 2006.

25. Bonifazi M, Sardella F, Lupo C. Preparatory versus main competitions: differences in performances, lactate responses and pre-competition plasma cortisol concentrations in elite male swimmers. Eur J Appl Physiol. 2000;82(5-6):368-73.

26. Fernandes RJ, Sousa M, Pinheiro A, Vilar S, Colaço, P, et al. Anaerobic threshold individualized assessment in a young swimmer. Open Sports Sci. J 2010;3(1).

27. de Bruijn R, Richardson M, Schagatay E. Increased erythropoietin concentration after repeated apneas in humans. Eur J Appl Physiol. 2008;102(5):609-13.

28. Richardson M, Bruijn RD, Holmberg HC, Björklund G, Haughey H, et al. Increase of hemoglobin concentration after maximal apneas in divers, skiers, and untrained humans. Can J Appl Physiol. 2005;30 (3):276-81.

29. Lemaitre F, Joulia F, Chollet D. Apnea: A new training method in sport? Med Hypotheses, 2010;74(3):413-5.

30. Stavrou V, Toubekis AG, Karetsi E. Changes in respiratory parameters and fin-swimming performance following a 16-week training period with intermittent breath holding. J Hum Kinet, 2015;49(1):89-8.

31. Schagatay E, van Kampen M, Emanuelsson S, Holm B. Effects of physical and apnea training on apneic time and the diving response in humans. Eur J Appl Physiol. 2000;82(3):161-9.

32. Joulia F, Steinberg JG, Faucher M, Jamin T, Ulmer C et al. Breath-hold training of humans reduces oxidative stress and blood acidosis after static and dynamic apnea. Respir Physiol Neurobiol. 2003;137(1):1927.

33. Davis FM, Graves MP, Guy HJ, Prisk GK, Tanner TE. Carbon dioxide response and breath-hold times in underwater hockey players. Undersea Hyperb. 1987;14(6):527-34.

34. Grassi B, Ferretti G, Costa M, Ferrigno M, Panzacchi A, et al. Ventilatory responses to hypercapnia and hypoxia in elite breath-hold divers. Respir Physiol. 1994;97(3):323-32.

35. Smith DJ, Norris SR, Hogg JM. Performance evaluation of swimmers. Sports Med. 2002;32(9):539-54.

36. Nygren-Bonnier M, Gullstrand L, Klefbeck B, Lindholm P. Effects of glossopharyngeal pistoning for lung insufflation in elite swimmers. Med Sci Sports Exerc. 2007;39(5):836-41.

37. Prommer N, Ehrmann U, Schmidt W, Steinacker JM, Radermacher P, et al. Total haemoglobin mass and spleen contraction: a study on competitive apnea divers, non-diving athletes and untrained control subjects. Eur J Appl Physiol. 2007;101(6):753-9.

38. Baković D, Eterović D, Saratlija-Novaković X, Palada I, Valic Z, et al. Effect of human splenic contraction on variation in circulating blood cell counts. Clin Exp Pharmacol Physiol. 2005;32(11):944-51.

39. Schagatay E, Haughey H, Reimers J. Speed of spleen volume changes evoked by serial apneas. Eur J Appl Physiol. 2005;93(4):447-52.

40. Seifert L, Chollet D, Chatard JC. Changes in coordination and kinematics during a 100-m front crawl. Med Sci Sports Exerc. 2007;10: 1784-93. 
41. Ferretti G. Extreme human breath-hold diving. Eur J Appl Physiol. 2001;84(4);254-71.

42. Viru AA, Viru M. Biochemical monitoring of sport training. Human Kinetics 2001.
43. Espersen K, Frandsen H, Lorentzen T, Kanstrup IL, Christensen NJ. The human spleen as an erythrocyte reservoir in diving-related interventions. J Appl Physiol. 2002;92(5):2071-9. 